\title{
Estimation of the Number of Persons in a Reverberant Environment Using Bistatic Radar
}

\author{
Marwan Yusuf*, Brecht De Beelde*, Emmeric Tanghe*, Eli De Poorter*, Luc Martens*, \\ Pierre Laly ${ }^{\dagger}$, Davy P. Gaillot ${ }^{\dagger}$, Martine Liénard ${ }^{\dagger}$, and Wout Joseph* \\ ${ }^{*}$ Ghent University-IMEC, Ghent, Belgium \\ ${ }^{\dagger}$ IEMN-TELICE, University of Lille, Lille, France \\ email: marwan.yusuf@ugent.be
}

\begin{abstract}
The theory of room electromagnetics provides a simple characterization of indoor microwave propagation. By considering the indoor environment as a lossy cavity, the exponential decay rate of the power-delay profile is related to the total absorption inside the room. In this paper, we explore the possibility of estimating the number of people inside a below-deck ship compartment using only the decay time constant, also known as reverberation time. First, we verify the reverberating nature of the room. Then, we find the relation between reverberation time and the number of people inside the room. We show that it is possible to estimate the number of people with a good accuracy, depending on the number of antennas used. With a success rate of $88 \%$, the estimation error is only 1 person when 16 spatially averaged antennas are used.
\end{abstract}

Index Terms-ships, propagation, measurements, room electronmagnetics, indoor environment, reverberation time, ACS, human detection

\section{INTRODUCTION}

Deploying wireless networks in confined, reflective spaces such as found in metallic warehouses, aircraft cabins, and below-deck compartments in ships is gaining lots of interest, mainly for automatic alarm, monitoring, and safety applications. In particular, below-deck spaces in ships have been a primary focus of a number of studies, which explored both RF propagation and wireless communication performance [1]-[5]. Current shipboard monitoring systems use extensive lengths of cables to connect a massive number of sensors to control units. Wired installation during ships construction results in a high cost and weight. In addition, ships represent a harsh environment in which wires are vulnerable to moisture, heat and toxic elements, making maintenance a very difficult task. On the other hand, wireless communication is a serious challenge in the particular environment of ships [5]. Such highly reflective environment is characterized by rich electromagnetic scattering, time and angular dispersion, and can exhibit features of a complex reverberant cavity. However, the method proposed in this paper uses a relatively simple propagation model with only a single parameter to describe the environment.

Similar to Room Acoustics [6], Room Electromagnetics theory views the indoor environment as a lossy cavity, where all the effective losses can be described by the exponentially decaying tail of the power-delay profile (PDP) [7], as shown in Fig. 1. The decay time constant, also known as reverberation

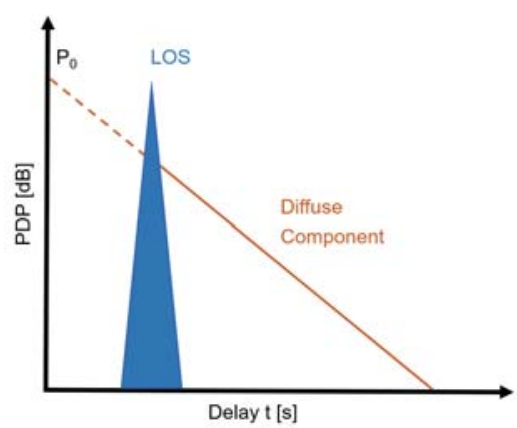

Fig. 1. Theoretical model of propagation including a LOS and an exponential tail with time constant $\tau$

time (RT), is a function of the volume and the absorption area of the room. The work in [8] validates the use of the acoustic reverberation models in electromagnetics by observing the reverberation time and gain, while altering the properties of the room in a controlled manner. It confirms that the reverberation is confined in the room where the transmitter and receiver are located. In [9], the impact of persons is studied by conducting measurements in a 24 passenger section of an aircraft cabin for the cabin being unoccupied and fully occupied. It shows that the total absorption from the seated passengers is dominated by the few who are near the transmitter. Also, the average absorption area of a passenger has been approximately determined at $2.4 \mathrm{GHz}$ for the fully occupied cabin. Experimental assessment of the absorption cross section and specific absorption rate of the human body is conducted in [10] at $2.3 \mathrm{GHz}$. The results confirm that the reverberation time is location and antenna independent by conducting the measurements in two different locations using two different measurement systems.

The contribution of this paper is to estimate the number of persons in a reverberant ship compartment based on room electromagnetics. Knowledge of the location or the movement of people on ships has a wide range of applications in commercial solutions and during the duty of state officials. Most solutions require the person to have a device that communicate with the network access points [11], [12]. The novelty of this work is based on detecting the presence of persons indoors without any active or passive components attached. Since the RT is related to the absorption area of the room, the presence 
TABLE I

MIMOSA CHANNEL SOUNDER SPECIFICATIONS

\begin{tabular}{lr}
\hline Parameter & Setting \\
\hline center frequency & $1.35 \mathrm{GHz}$ \\
bandwidth & $80 \mathrm{MHz}$ \\
number of Tx and Rx antennas & 8 \\
Tx and Rx polarization & $\mathrm{H} / \mathrm{V}$ \\
number of OFDM subcarriers & 6560 \\
OFDM symbol duration $T_{S}$ & $81.92 \mu \mathrm{s}$ \\
cyclic prefix duration $T_{C P}$ & $0 \leq T_{C P} \leq T_{S}$ \\
$16 \times 16$ channel acquisition time & $2\left(T_{S}+T_{C P}\right) \leq 327.68 \mu \mathrm{s}$
\end{tabular}

of people in the room will alter the total absorption area, and hence, the RT. To this end, the PDP is measured in a belowdeck room using a channel sounder, first without the presence of humans. Then, persons are introduced into the room one at a time. Eventually, the RT is calculated by means of linear regression and for each case, the number of people inside the room is estimated using the theory of room electromagnetics.

The outline of this paper is as follows. The measurement scenario is described in Section II. Section III describes the methodology to extract the RT, while in Section IV results of the measurements and RT are presented. Section V shows the estimation results for the number of people, and conclusions are drawn in Section VI.

\section{Measurement Configuration And Scenario}

Channel measurements are performed with the MIMOSA radio channel sounder [13]. The center frequency is $1.35 \mathrm{GHz}$ and the transmission bandwidth is $80 \mathrm{MHz}$. The sounder uses dual-polarized patch antenna arrays with horizontal $(\mathrm{H})$ and vertical (V) polarization. For this measurement campaign, 8element rectangular arrays are used at both the transmitter (Tx) and receiver $(\mathrm{Rx})$. Orthogonal frequency division multiplexing (OFDM) is used to encode 8 parallel transmit channels, and by connecting each to a two-port RF switch for the two polarizations, a total of $16 \times 16$ channels are measured. The channel sounder is fully parallel: the data from each transmit antenna is simultaneously modulated onto the carriers using interleaved frequency division multiple access. Table I summarizes the technical specifications of MIMOSA.

The measurement campaign is carried out in the steering gears room of a bulk carrier vessel, shown in Fig. 2. The Tx and Rx of the channel sounder are placed inside the room and the door is kept closed during the measurement. The room has a height of $4 \mathrm{~m}$ and a volume of $600 \mathrm{~m}^{3}$, approximately. The antenna array of $\mathrm{Rx}$ is fixed at $2 \mathrm{~m}$ height, with radiation towards the corner of the room [1]. The reverberating nature of the room is first investigated by measuring the radio channel at random locations inside the room. This is done by placing the Tx antenna array at 6 different locations. Then, the Tx is fixed and the radio channel is recorded for different numbers of people inside the room, ranging from 0 to 6 persons at different locations. The channel sounder with fixed Tx and Rx, thus, works as a bistatic radar. Each channel is measured 200 times in static conditions and averaged to reduce measurement

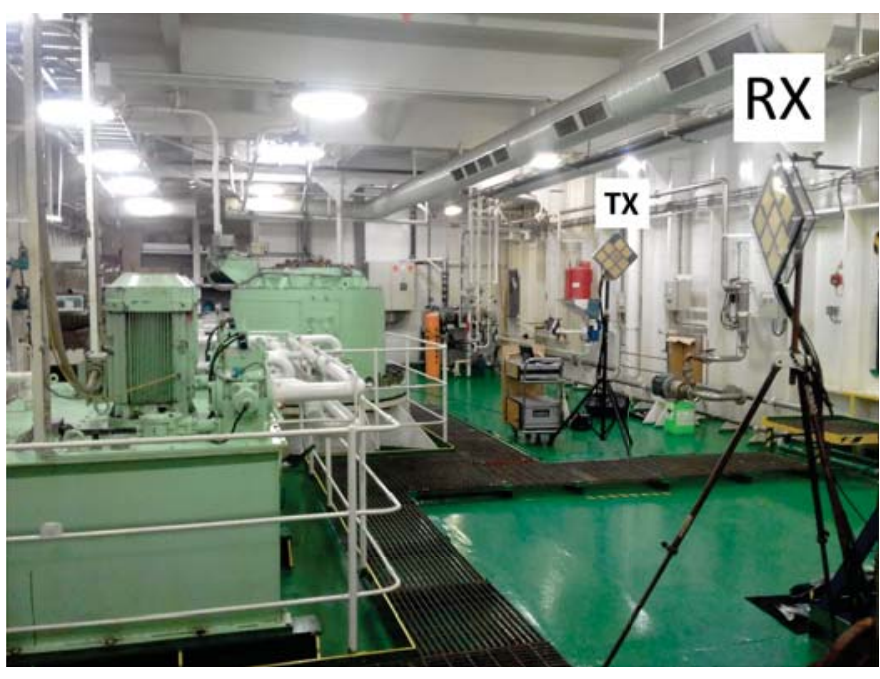

Fig. 2. Below-deck ship compartment with rectangular antenna arrays of the channel sounder

noise. Hence, a total of $\mathrm{S}=7 \times 64 \times 4$ PDPs are calculated, where the value 64 results from the $8 \times 8$ antennas, and 4 from the polarizations (VV, HV, VH, HH) with the first for Tx and the second for $\mathrm{Rx}$.

\section{Methodology}

In order to estimate the number of people inside the room, we first need to extract the RT. In indoor propagation, multiple reflections and scattering lead to an exponential decay of power with a decay constant $\tau$. The diffuse scattering model in Fig. 1 can be represented as

$$
P(t)=P_{0} \exp (-t / \tau)
$$

where $t$ is the arriving time of a point on the delay axis and $P(t)$ is the corresponding power. The RT $\tau$ can be expressed as [7]

$$
\tau=\frac{4 V}{c A_{n}}
$$

where $V$ is the room volume, $A_{n}$ is the total absorption area and $c$ is the velocity of light. In a fixed environment, where the contents of the room do not change except for the number of people inside, the total absorption area can be written as

$$
A_{n}=A_{0}+n \times A C S
$$

where $A_{0}$ is the absorption area of the room without people, $n$ is the number of people in the room and $A C S$ is the average absorption cross section area of the human body. Each additional person in the room increases the total absorption area by the amount of the $A C S$ of the human body. From the RT in (2), it is possible to assess the absorption area $A_{n}$, and assuming the $A C S$ is known, the number of people $n$ can be calculated from (3).

For the extraction of the reverberation time, the slope of the PDP exponential decay has to be calculated. However, the 


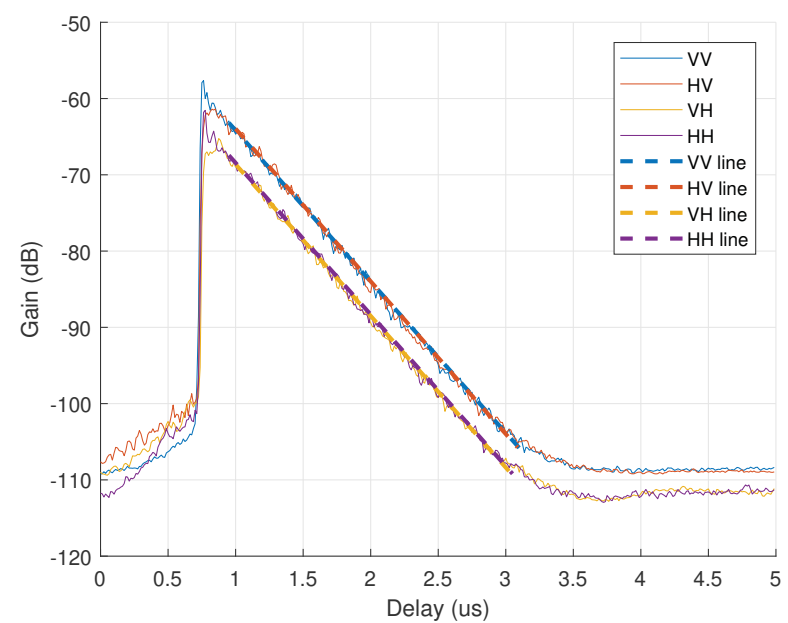

Fig. 3. PDP and linear regression for different polarizations in case of an empty room

tail of the PDP is not perfectly linear due to noise, LOS and specular components. A least-square regression line is thus used to fit the PDP over a delay range, and the choice of this range is done automatically. First, to avoid LOS, the starting point is taken as the mean arrival time $T_{m}$ given by

$$
T_{m}=\frac{\sum t P(t)}{\sum P(t)}
$$

Second, the noise level is calculated by averaging all the power values with large delays from the PDP where no multipath components above the noise are expected. Finally, in order to avoid the effect of the noise floor, the ending point of the regression delay range is taken when the power level reaches $5 \mathrm{~dB}$ above the calculated noise level.

\section{EXPERIMENTAL RESUlTS AND POSTPROCESSING}

\section{A. RT Extraction}

In order to investigate the reverberating nature of the environment, the RT is calculated at 6 different locations in case of an empty room. The PDP is first spatially averaged over all the antennas before extracting the RT, to reduce small-scale fading. Fig. 3 shows the PDP at one location for different polarizations in case of an empty room. The figure shows the identical diffuse power level of the co-polar and cross-polar channels, suggesting that the complex field in the room is completely random and the environment is indeed reverberating. To further strengthen that assumption, the RT is calculated at 6 random locations in the room. Fig. 4 shows the RT at each location for different polarizations. It is clear that the RT value is independent of the location as well as the polarization of the antennas, with a relative standard deviatoin of $0.99 \%$. This verifies the reverberating nature of the room, in accordance with [10].

In the same way, the RT is calculated when the room is occupied by people, starting from 1 till 6 persons. Fig. 5 shows

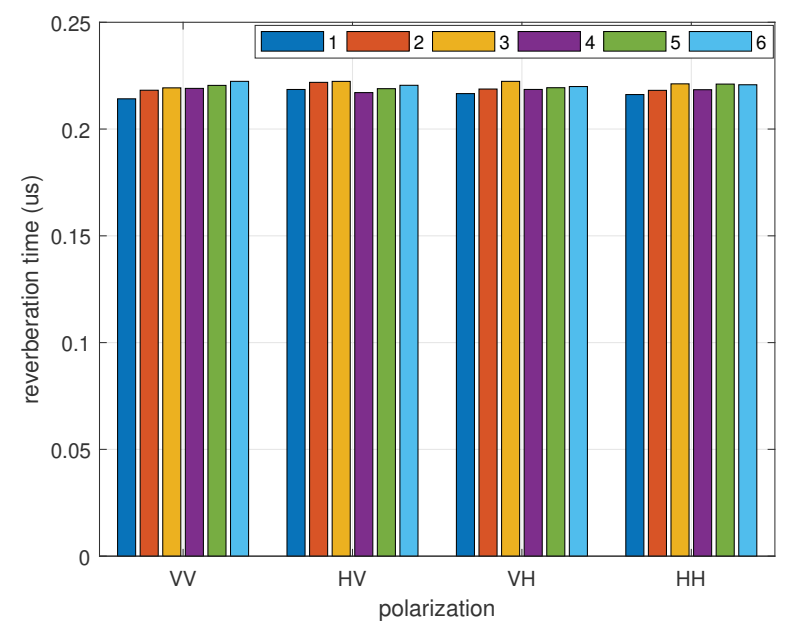

Fig. 4. RT at 6 locations for differemt polarizations in case of an empty room, where the first polarization $(\mathrm{H} / \mathrm{V})$ is for $\mathrm{Tx}$ and the second one is for $\mathrm{Rx}$

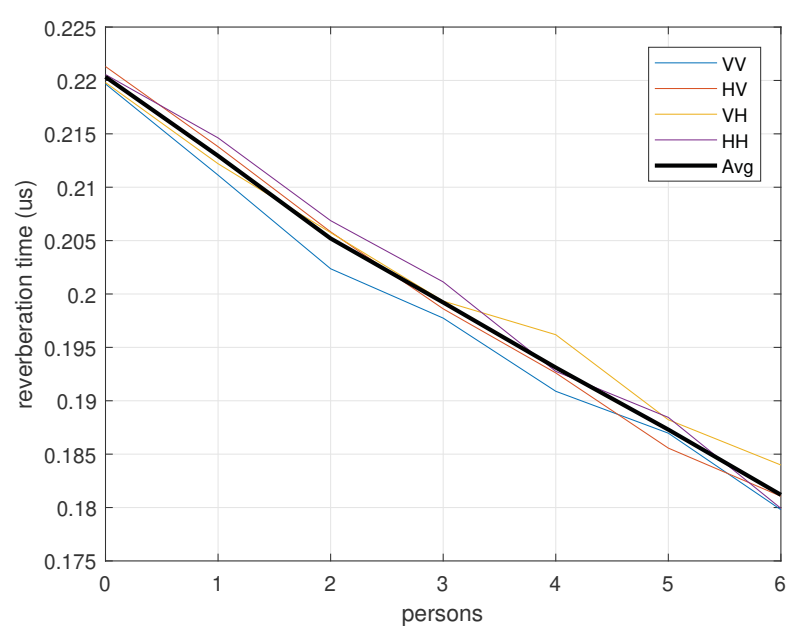

Fig. 5. RT versus the number of persons inside the room for different polarizations

the RT versus the number of persons for different polarizations. The figure clearly indicates that the RT is inversely proportional to the number of persons in an almost linear way. This validates the relation between the RT, absorption area, and the number of persons in the room for different polarizations. Fig. 5 also shows that the RT for VV is slightly lower than the average of all polarizations, suggesting that human bodyis more prone to absorbing vertically polarized compared to horizontally polarized waves. Nevertheless, the RT for different polarizations are similar enough (relative standard deviation of $0.61 \%$ ) to aggregate them into a larger sample size.

\section{B. Absorption Area and ACS}

Since our method assumes the knowledge of the average $A C S$ of the persons inside the room, it would be of interest 
TABLE II

PARAMETERS CALIBRATION IN $\left(\mathrm{M}^{2}\right)$

\begin{tabular}{ccc}
\hline & Full data & Calibration set $(20 \%)$ \\
\hline$A C S$ & 1.33 & 1.26 \\
$A_{0}$ & 36.44 & 36.57 \\
\hline
\end{tabular}

to first calculate the $A C S$ based on our measurements and compare it to prior studies. From (3), the $A C S$ is the slope of the linear regression of different absorption areas $A_{n}$ related to a different number of persons ( $n=0,1, . ., 6)$. The calculation of $A_{n}$ is given in (2) by averaging the PDPs of all polarizations before extracting the RT $\tau_{n}$. This gives an $A C S$ of $1.3 \mathrm{~m}^{2}$. Previous studies indicate an $A C S$ of 0.3-0.4 $\mathrm{m}^{2}$ for the given frequency [14]. A reason for this deviation is the complex structure of the room, making the calculation of the exact volume a difficult task. Moreover, (2) assumes homogeneous repartition of energy within the room, and consequently uniformly distributed absorption, and that the field is completely diffuse [7]. In general, the field will be sufficiently diffuse if the basic dimensions of the room are almost the same, walls are not parallel, and most absorbing surfaces are divided into parts and uniformly distributed. In practice, almost none of these requirements are fulfilled.

Nonetheless, to be able to estimate the number of people from the RT, the $A C S$ and $A_{0}$ of our scenario need to be calibrated. This is done by randomly splitting the measurement data S into two sets: a calibration set of $20 \%$ of the data for calculating the $A C S$ and $A_{0}$, and a testing set of $80 \%$ to actually estimate the number of people based on the calibrated values. Table II summaries the calculated calibration values from both the full data set and the calibration set. The small difference between the values of the two sets indicates how well the estimation performance is expected to be.

\section{ANALYSIS AND DISCUSSION}

In this section, the number of persons inside the room is estimated. Table II lists the calibrated $A C S$ and $A_{0}$ values, while the total absorption area $A_{n}$ is calculated based on the measured $\tau$ from (2) for each case. From (3), the estimate number of persons is obtained as

$$
\widehat{n}=\left[\frac{A_{n}-A_{0}}{A C S}\right]
$$

where a simple round operator [.] is used to get the integer estimate. The estimation performance depends on the RT calculation as aforementioned. For a more accurate RT, spatial averaging of the PDPs from several antenna elements is used. Fig. 6 presents the estimation performance for different number $m$ of averaged PDPs from the testing set. It shows the histogram of the estimation error $e$ defined as the absolute difference between the estimate and the actual number of persons

$$
e=|n-\widehat{n}|
$$

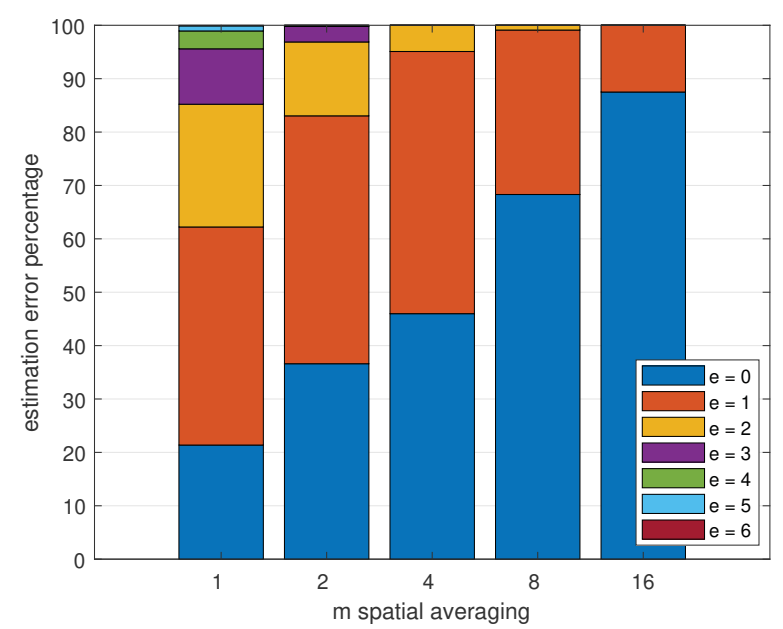

Fig. 6. Estimation error ( $\mathrm{e}=0-6$ persons $)$ histogram of the number of persons in the room for different spatial averaging sizes

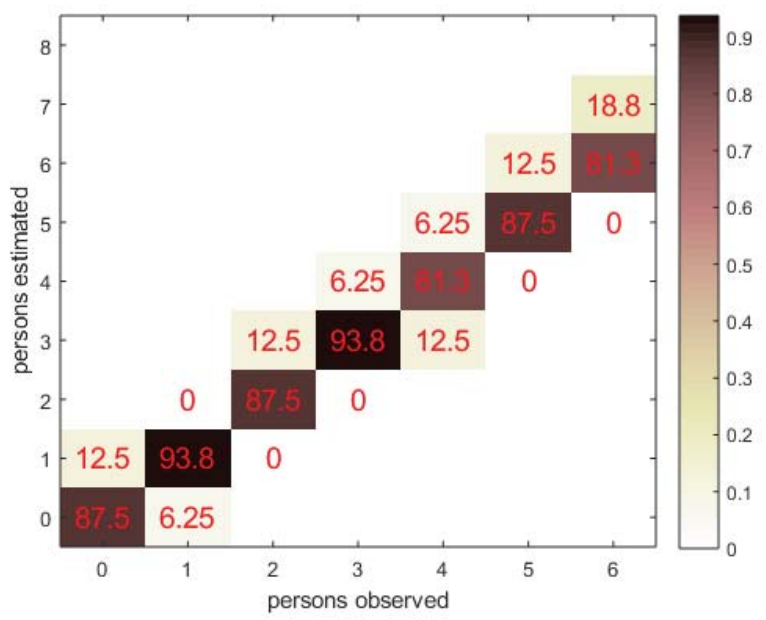

Fig. 7. Confusion matrix for the estimation percentage of the number of persons in the room for $m=16$

With only a single antenna $(m=1)$, the estimation error can reach up to 6 persons with an estimation success rate of only $21.4 \%$. As $m$ increases, the estimation performance improves in terms of higher success rate and smaller number of persons as estimation error. With 16 spatially averaged links, the success rate is $88 \%$ with only 1 -person error of $12 \%$. Fig. 7 shows the confusion matrix for $m=16$. It gives the details of the estimation percentage per each case of observed number of persons. This clearly shows the good performance of the estimation algorithm, where all the cases have a success rate above $81 \%$, and all the estimation error larger than 1-person is $0 \%$.

It should be acknowledged that, while the persons may not be exposed identically, this method assumes they are, as shown in (3), which influences the estimation performance. Factors that can improve the performance of estimation in- 
clude: increasing the sample size of measurement scenarios, and increasing the number of antennas or spatial averaging $m$ for improved calculation of the RT, and hence, improved overall estimation performance.

\section{CONCLUSION}

Based on the simple theory of Room Electromagnetics, this paper explores the feasibility of estimating the number of persons inside a reverberant ship compartment by means of only measuring the reverberation time. Our findings indicate that there is indeed an inverse relation between the number of persons inside the room and the reverberation time. We observe that the reverberation time is the same, independent of the antenna or the location used for measurement inside the room. A calibration of the ACS is needed before performing the estimation, which is done via measurements. While the estimation performance is very low in case of a single antenna, it can be enhanced via spatial averaging of multiple antennas. With 16 spatially-averaged PDPs, a good estimation performance is achieved with only a 1-person error of $12 \%$.

\section{ACKNOWLEDGMENT}

This work is part of the Internet-of-Shipping (IoS) research project. IoS is co-funded by IMEC and Flanders Innovation \& Entrepreneurship. The authors would like to thank Ivan Renette from Exmar ship management for facilitating the measurement campaign.

\section{REFERENCES}

[1] G. B. Tait and M. B. Slocum, "Electromagnetic environment characterization of below-deck spaces in ships," in 2008 IEEE International Symposium on Electromagnetic Compatibility. IEEE, 2008, pp. 1-6.

[2] G. Tait, M. Slocum, and R. Richardson, "On multipath propagation in electrically large reflective spaces," IEEE Antennas and Wireless Propagation Letters, vol. 8, pp. 232-235, 2009.

[3] A. Mariscotti, M. Sassi, A. Qualizza, and M. Lenardon, "On the propagation of wireless signals on board ships," in 2010 IEEE Instrumentation \& Measurement Technology Conference Proceedings. IEEE, 2010, pp. $1418-1423$
[4] K. Wanuga, R. Measel, C. S. Lester, D. J. Bucci, D. Gonzalez, R. Primerano, M. Kam, and K. R. Dandekar, "Performance evaluation of MIMO OFDM systems in on-ship below-deck environments," IEEE Antennas and Wireless Propagation Letters, vol. 13, pp. 173-176, 2014.

[5] R. Measel, C. S. Lester, D. J. Bucci, K. Wanuga, G. Tait, R. Primerano, K. R. Dandekar, and M. Kam, "An empirical study on the performance of wireless OFDM communications in highly reverberant environments," IEEE Transactions on Wireless Communications, vol. 15, no. 7, pp. 4802-4812, 2016.

[6] H. Kuttruff, Room acoustics. Crc Press, 2016.

[7] J. B. Andersen, J. Nielsen, G. Pedersen, G. Bauch, and J. Herdin, "Room electromagnetics," IEEE Antennas and Propagation Magazine, vol. 49, no. 2, pp. 27-33, 2007

[8] G. Steinböck, T. Pedersen, B. H. Fleury, W. Wang, and R. Raulefs, "Experimental validation of the reverberation effect in room electromagnetics," IEEE Transactions on Antennas and Propagation, vol. 63, no. 5, pp. 2041-2053, 2015

[9] J. B. Andersen, K. L. Chee, M. Jacob, G. F. Pedersen, and T. Kurner, "Reverberation and absorption in an aircraft cabin with the impact of passengers," IEEE Transactions on Antennas and Propagation, vol. 60, no. 5, pp. 2472-2480, 2012.

[10] A. Bamba, W. Joseph, J. B. Andersen, E. Tanghe, G. Vermeeren, D. Plets, J. Ø. Nielsen, and L. Martens, "Experimental assessment of specific absorption rate using room electromagnetics," IEEE Transactions on Electromagnetic Compatibility, vol. 54, no. 4, pp. 747-757, 2012.

[11] K. Witrisal, P. Meissner, E. Leitinger, Y. Shen, C. Gustafson, F. Tufvesson, K. Haneda, D. Dardari, A. F. Molisch, A. Conti et al., "Highaccuracy localization for assisted living: $5 \mathrm{G}$ systems will turn multipath channels from foe to friend," IEEE Signal Processing Magazine, vol. 33, no. 2, pp. 59-70, 2016.

[12] P. Rajchowski, K. K. Cwalina, J. Sadowski, and J. Stefański, "Hybrid system for a person tracking in harsh indoor environments," in 2019 European Navigation Conference (ENC). IEEE, 2019, pp. 1-6.

[13] P. Laly, D. P. Gaillot, M. Liénard, P. Degauque, E. Tanghe, W. Joseph, and L. Martens, "Flexible real-time MIMO channel sounder for multidimensional polarimetric parameter estimation," in Antenna Measurements \& Applications (CAMA), 2015 IEEE Conference on. IEEE, 2015, pp. $1-3$.

[14] I. Flintoft, M. Robinson, G. Melia, A. Marvin, and J. Dawson, "Average absorption cross-section of the human body measured at $1-12 \mathrm{GHz}$ in a reverberant chamber: results of a human volunteer study," Physics in Medicine \& Biology, vol. 59, no. 13, p. 3297, 2014. 